\title{
A Hydro-morphodynamic Numerical Model of a Wadden Sea Area
}

\author{
E. Falke, T. Albers, E. Pasche
}

INTRODUCTION

Based on field measurements a numerical model is developed to improve the knowledge of high resoluted morphodynamic processes in shallow water sections of Wadden Sea areas.

Supported by the Hamburg Port Authority (HPA) the Institute of River and Coastal Engineering at the Hamburg University of Technology (TUHH) ran extensive measurements to collect dat in the Wadden Sea area "Neuferder Watt" in the mouth of the (fig. 1). Between July 2006 and September 2009 water levels, flow and wave parameters were recorded in high resolution as well as the concentrations of suspended sediment. The bathymetry of the main tidal channel "Neufelder Rinne" was determined by a multi-beam echo sounder in frequent intervals.

INTVESTIGATIONAREA

The Neufelder Watt with the "Neufelder Sand" is a sandflat in the mouth of the estuary Elbe (fig. 1). The area is in close interaction with the main stream of the estuary. The average tidal range is about three meters.

The tidal flats around the main tidal channel "Neufelder Rinne" fall dry during a longer period around low tide. The Neufelder Rinne is of high importance for a huge part of the Neufelder Watt due to its dominating role for the drainage and watering of the tidal flats. There are rapid morphodynamic changes in this Wadden Sea area especially at the inlet of the creek which are investigated by ALBERS ET AL.(ALBERS ET AL., 2009).

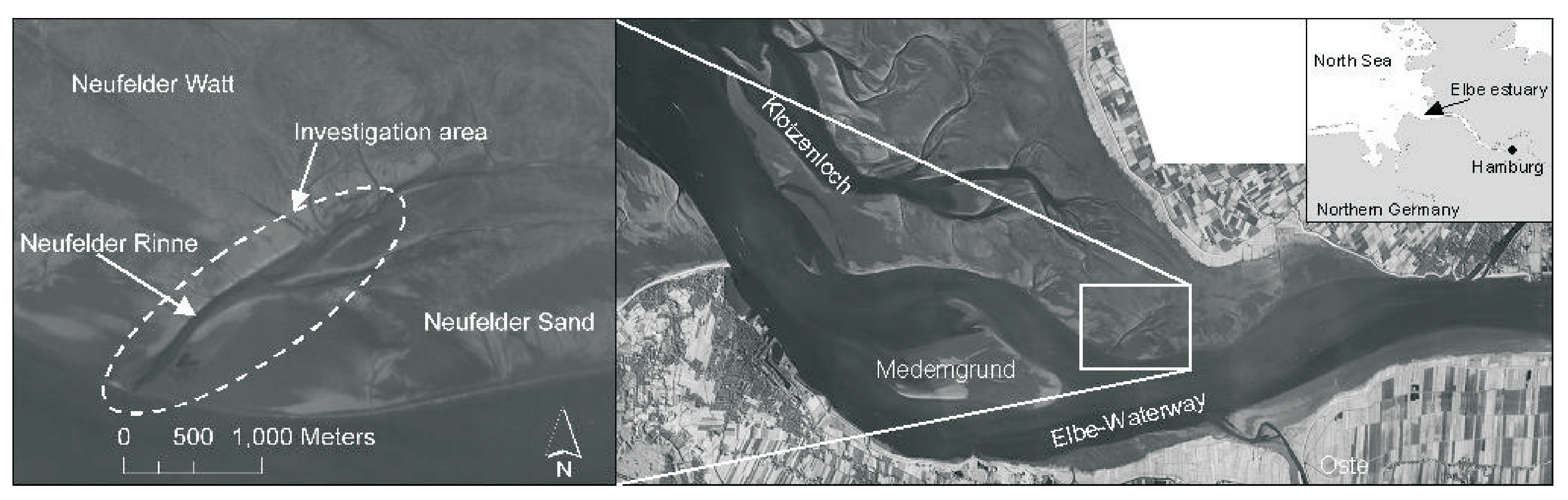

Fig. 1: Investigation area in the mouth of the estuary Elbe at the German North Sea Coast

METHOD

The application of numerical models demands the allocation of initial and boundary conditions. To capture the flow and sediment transport phenomena in Wadden Sea areas a high spatial resolution is necessary. The main challenge is the definition of the boundary conditions: beside the waterlevels discharges are the driving forces for hydrodynamic numerical models but they can not be measured in the Wadden Sea area because of the permanent changes of the flow situation due to the tide. The method to define the hydrodynamic situations of the investigation area is to set up two seperate numerical models: a larger surrounding large scale hydrodynamic model of the estuary mouth is developed to generate the hydrodynamic boundary conditions for a detailed small scale hydromorphodynamic model which covers only a part of the main tidal channel and the surrounding tidal flats (fig. 2 and fig. 3). The transfer of the boundary conditions between the different scaled models is just oneway, there is no feedback from the detailed model to the surrounding model.

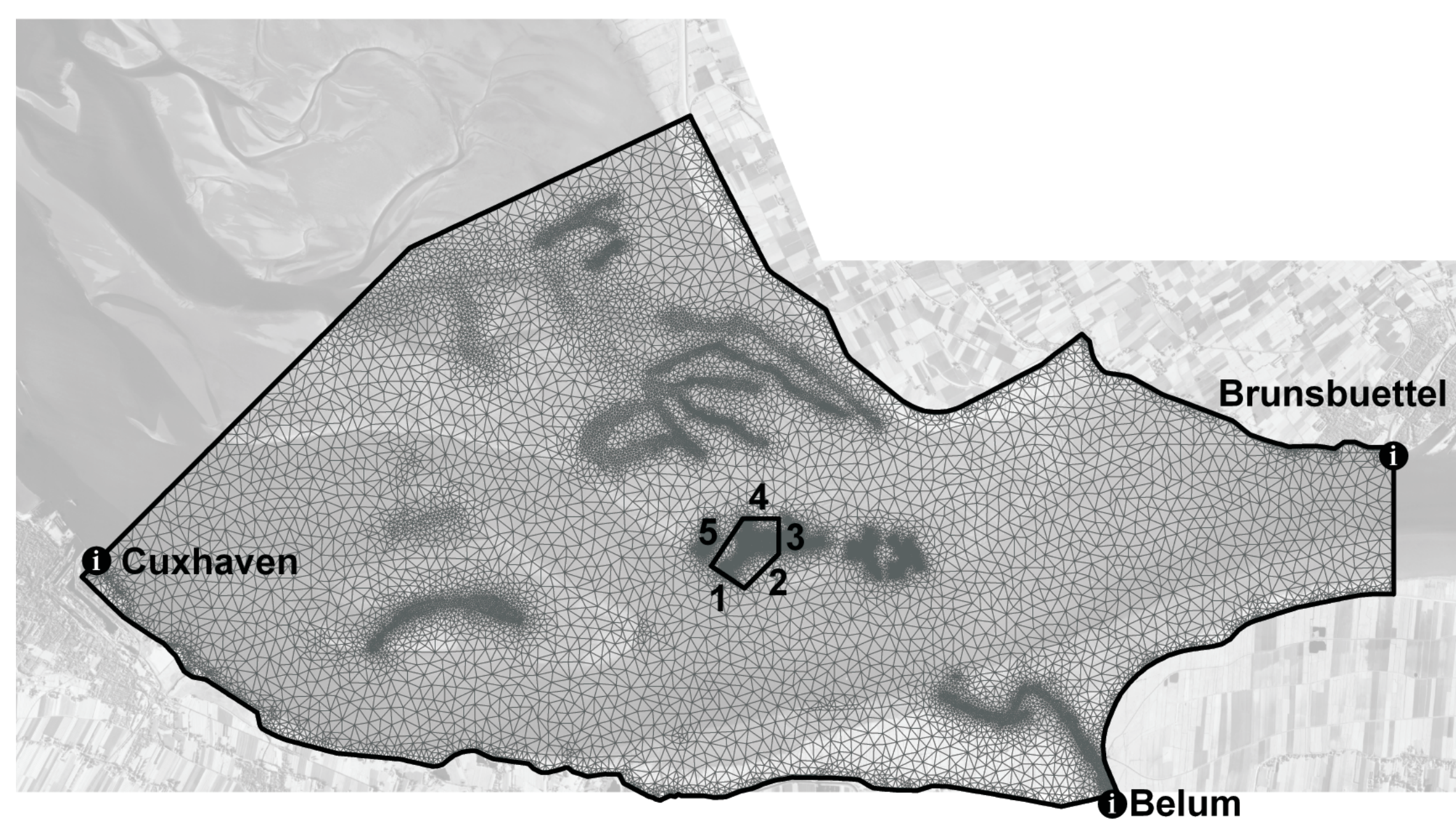

Fig. 2: Finite Element mesh of the larger surrounding model and the

NUMERICAL MODEL

The Finite Element Model RMA.Kalypso is used for the two dimensional depth averaged numerical modelling of the hydrodynamic within the mouth of the estuary Elbe as well as for the hydromorphodynamic modelling of the Neufelder Watt.

The larger surrounding model covers an area of about $220 \mathrm{~km}^{2}$ by using nearly 35,000 irregular triangular Finite Elements. Only the investigation area and the for the flooding and drainage of the tidal flat areas important tideways are discretised with high resolution. The detailed model discretises an area of only $1.3 \mathrm{~km}^{2}$ with a structured grid of quadratic Finite Elements with an edge length of $10 \mathrm{~m}$. The large scale model is controlled by the measured water levels of the gauging stations Brunsbuettel, Belum and Cuxhaven, whereas the small scale model is controlled by four discharges (boundary line 2 to 5 ) and one water stage (line 1 ) which are generated inside the larger surrounding model (fig 2). The recorded sediment concentrations within the investigation area are used as boundary conditions to model the sediment transport within the detailed model. Therefore the suspended sediment transport is calculated using the van Rijn sediment transport formula (1993) which considers not only the sediment properties, the waterdepth and the flow velocity but also wind and waves. Afterwards the advection-diffusion equation is solved in the equation system and the bed evolution is subsequently calculated within a balance equation.

SIMULATION

The calibration of the hydrodynamic models focuses on the drying an rewetting of the tidal flats and the accuracy of the simulated hydrodynamic parameters. The Marsh Porosity option is used which considers a subsurface flow similar to the groundwater flow and integrates it to the surface flow level of discretisation. The tidal flats run dry at low tide as expected (fig. 5).
After the following hydrodynamic validation based on different flow situations the sediment transport in the detailed model is calibrated and validated as well. Scenarios with a time range of severa weeks in the years 2007 and 2008 are modelled subsequently to verify whether the observed morphodynamic processes can be modelled numerically.

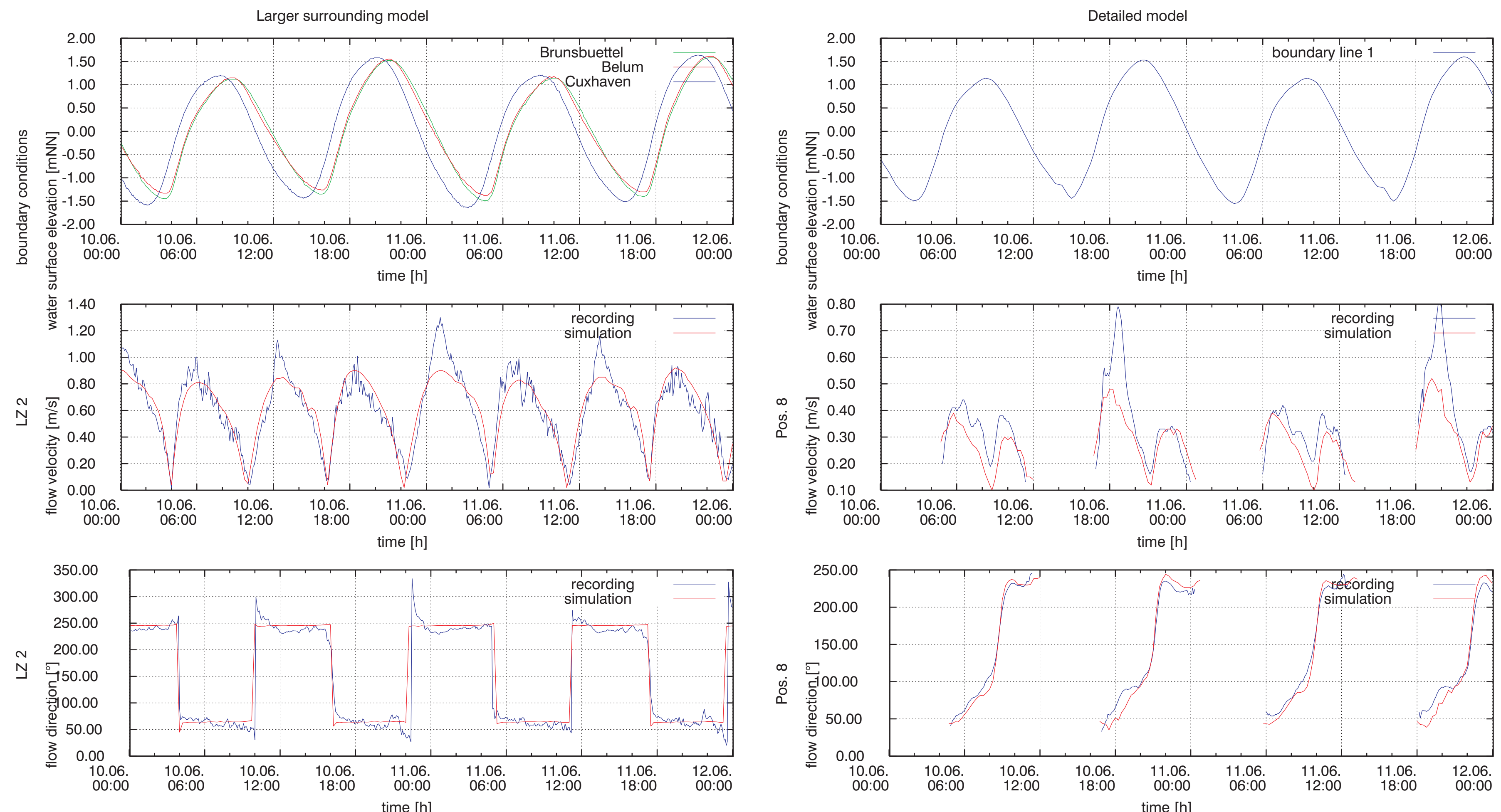

Fig. 4: Simulated flow velocities and directions compared to the
June 2007

RESULTS

The hydrodynamic of both numeric models is calibrated and validated by comparing the simulated water levels and flow parameters with the measured data at the gauging stations of the modelled areas (fig. 4). Afterwards the selected flow scenarios are simulated.

The calculated water levels are nearly identical with the recorded ones at all gauging stations. The flow reversal is very well approximated by the model and so is the flow velocity. Only the peaks are not depicted which is caused by the chosen calculation time step of 15 minutes. Fig. 4 illustrates the norforman 0 of at position 8 and at 12.

The computed morphodynamic changes within the detailed model are compared to the measured differences of the bathymety in the Neufelder Rinne for different periods of time. The results fit qualitative as fig 6 shows exemplarily for a two week scenario in spring 2008.

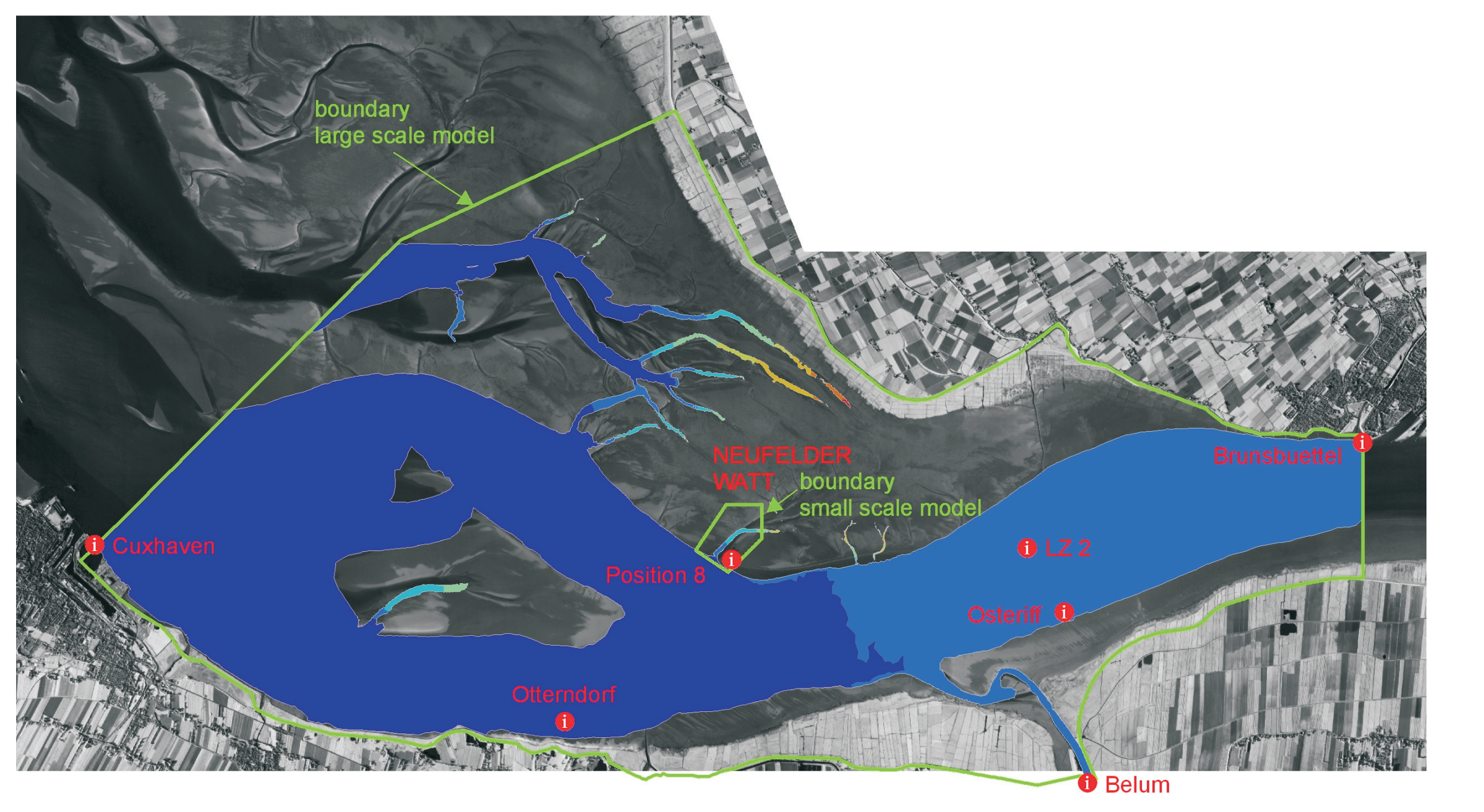

Fig. 5: Model boundaries, positions of gauging stations and simulated water

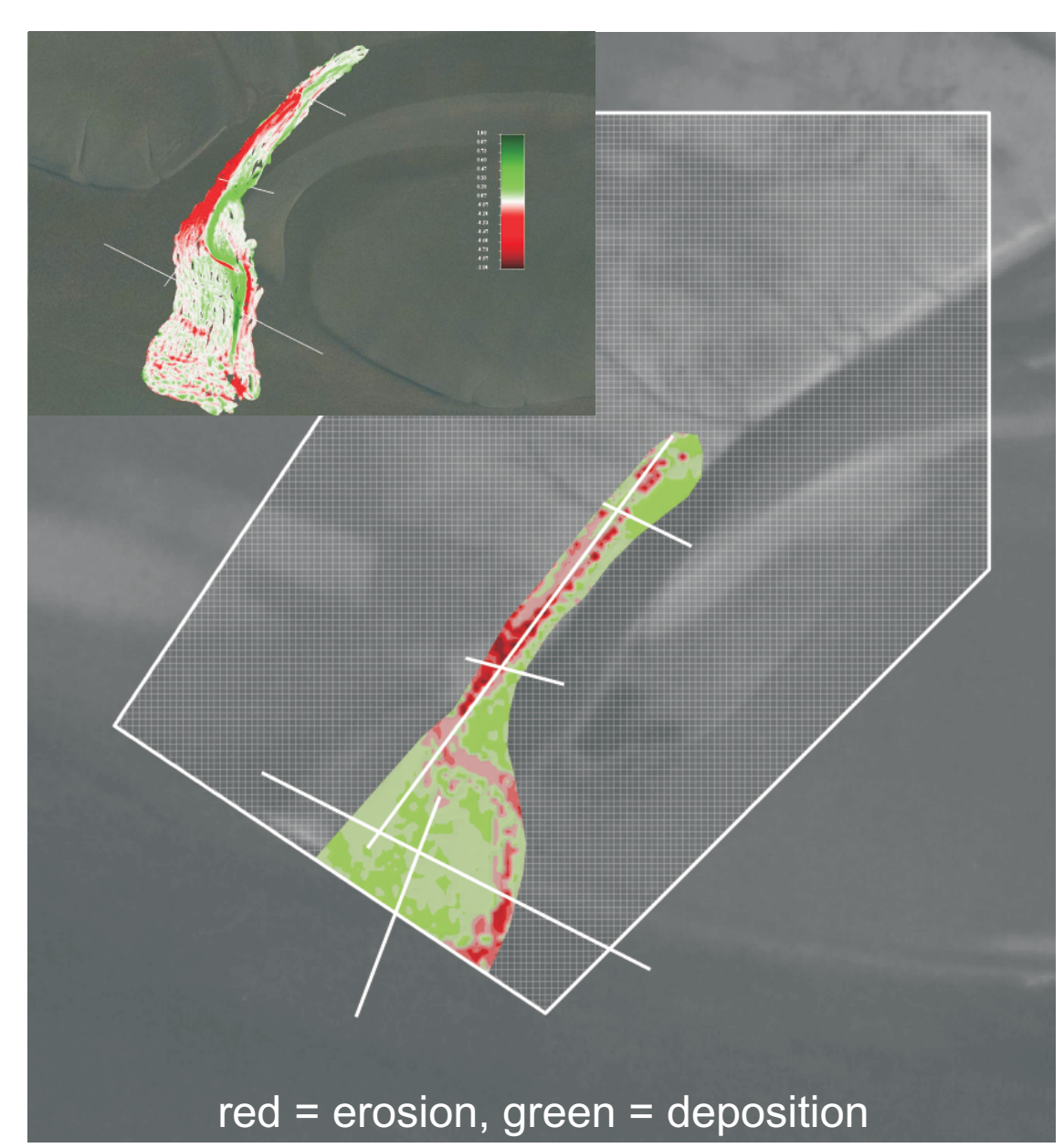

Fig. 6: Measured (top) and simulated 23.04.2008-06.05.2008
CONCLUSIONS

The definition of the detailed hydrodynamic flow situation of the Wadden Sea area Neufelder Watt is successfully done by setting up a larger surrounding model to generate the hydrodynamic boundary conditions for the detailed small scale hydro-morphodynamic model of the investigation area as the simulated water levels and especially the flow parameters corresponds very well to the recorded ones (fig. 4). The drying and rewetting of the tidal creeks is successfully simulated by using the Marsh porosity option. The dewatering of the tidal flats via the tidal creeks requires an accurate discretisation of the surface elevation so that a realistic flow field can occur (fig. 5).

The numerical modelling of the sediment transport within the detailed model by using the generated hydrodynamics as well as the recorded suspended load concentration, wind and waves as boundary conditions does work out as the modelled morphodynamic changes are fitting with the measured ones (fig. 6).

OUTLOOK

There will be more simulations of morphodynamic changes for different flow scenarios at which the bathymetry of the Neufelder Rinne is known at the starting time and at the finishing time. All results will then be compared with the field measurements to verify whether the observed small scale morphodynamic processes can be modelled numerically and if there are any restrictions due to the limits of the sediment transport model or the spatial resolution which neglects physical processes with a smaller dimension than $10 \mathrm{~m}$ in horizontal direction.

REFERENCES

Albers, T, von Lieberman, N. and FALKe, E., 2009, Morphodynamic Processes on Tidal Flats in Estuaries, ICS 2009 (Proceedings), In: Journal of Coastal Research, Special Issue 56, Lisbon, Portugal, ISSN 0749-0258, 1325-1329

KING, I. P., 2006, A Finite Element Model for Stratified Flow and Cohesive Sediment/Sand

Transport - RMA-10 Users Guide Version 3.5D, Sydney, Australia
Dipl.-Ing. Eva Falke

Phone: +49-40-42878 3067

E-mail: eva.falke@tuhh.de
Research Associate

Hamburg University of Technology Institute of River and Coastal Engineering www.tuhh.de/wb 